\title{
Swim Pressure: Stress Generation in Active Matter
}

\author{
S. C. Takatori, W. Yan, and J. F. Brady ${ }^{*}$ \\ Division of Chemistry and Chemical Engineering, California Institute of Technology, \\ Pasadena, California 91125, USA
}

(Received 24 February 2014; published 11 July 2014)

\begin{abstract}
We discover a new contribution to the pressure (or stress) exerted by a suspension of self-propelled bodies. Through their self-motion, all active matter systems generate a unique swim pressure that is entirely athermal in origin. The origin of the swim pressure is based upon the notion that an active body would swim away in space unless confined by boundaries - this confinement pressure is precisely the swim pressure. Here we give the micromechanical basis for the swim stress and use this new perspective to study self-assembly and phase separation in active soft matter. The swim pressure gives rise to a nonequilibrium equation of state for active matter with pressure-volume phase diagrams that resemble a van der Waals loop from equilibrium gas-liquid coexistence. Theoretical predictions are corroborated by Brownian dynamics simulations. Our new swim stress perspective can help analyze and exploit a wide class of active soft matter, from swimming bacteria to catalytic nanobots to molecular motors that activate the cellular cytoskeleton.
\end{abstract}

DOI: 10.1103/PhysRevLett.113.028103

From flocks of animals and insects to colonies of living bacteria, so-called active matter exhibits intriguing phenomena owing to its constituents' ability to convert chemical fuel into mechanical motion. Active matter systems generate their own internal stress, which drives them far from equilibrium and thus frees them from conventional thermodynamic constraints, and by so doing they can control and direct their own behavior and that of their surrounding environment. This gives rise to fascinating behavior such as spontaneous self-assembly and pattern formation [1-3] but also makes the theoretical understanding of their complex dynamical behaviors a challenging problem in the statistical physics of soft matter.

In this Letter we have identified a new principle that all active matter systems display-namely, through their selfmotion they generate an intrinsic swim stress that impacts their dynamic and collective behavior. In contrast to thermodynamic quantities such as the chemical potential and free energy, the mechanical pressure (or stress) is valid out of equilibrium because it comes directly from the micromechanical equations of motion. To motivate this new perspective, we focus on the simplest model of active matter-a suspension of self-propelled spheres of radii $a$ immersed in a continuous Newtonian solvent with viscosity $\eta$. The active particles translate with a constant, intrinsic swim velocity $\boldsymbol{U}_{0}$ and tumble with a reorientation time $\tau_{R}$. We do not include the effects of hydrodynamic interactions among the particles.

The origin of the swim pressure is based upon a simple notion-a self-propelled body would swim away in space unless confined by boundaries. The pressure exerted by the surrounding walls to contain the particle is precisely the swim pressure. This is similar to the kinetic theory of gases,
PACS numbers: 47.63.mf, 05.65.+b, 47.63.Gd, 64.75.Xc

where molecular collisions with the container walls exert a pressure. Although it is clear that such a swim pressure should exist, what is its micromechanical origin, and how is it to be explained and expressed in basic physical quantities?

The swim pressure is the trace of the swim stress, which is the first moment of the self-propulsive force,

$$
\boldsymbol{\sigma}^{\text {swim }}=-n\left\langle\boldsymbol{x} \boldsymbol{F}^{\mathrm{swim}}\right\rangle,
$$

where $n$ is the number density of active particles, $\boldsymbol{x}$ is the absolute position, and $\boldsymbol{F}^{\text {swim }} \equiv \zeta \boldsymbol{U}_{0}$ where $\zeta$ is the hydrodynamic resistance coupling translational velocity to force

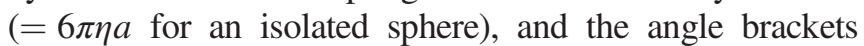
denote an average over all particles and over time. It is permissible for determining the stress to interpret the selfpropulsion of an active particle as arising from a swim force, $\boldsymbol{F}^{\text {swim }}$ (see below). As a result, $-\left\langle\boldsymbol{x} \boldsymbol{F}^{\text {swim }}\right\rangle$ gives a singleparticle self contribution to the stress. (This distinguishes Eq. (1) from the familiar $-\left\langle\boldsymbol{x}_{i j} \boldsymbol{F}_{i j}\right\rangle$ form seen in classical analyses of molecular liquids, where subscripts $i j$ indicate pairwise interactions [4]). Equation (1) comes from the virial theorem [5], which expresses the stress (or pressure) in terms of the forces acting on a system: $\boldsymbol{\sigma}=-1 / V\left\langle\sum_{i}^{N} \boldsymbol{x}_{i} \boldsymbol{F}_{i}\right\rangle$. In Eq. (1) we take the forces $\boldsymbol{F}_{i}$ to be the swim force of each particle, $\boldsymbol{F}^{\text {swim }}$.

The position of a particle at time $t$ is $\boldsymbol{x}(t)=\int \boldsymbol{U}\left(t^{\prime}\right) d t^{\prime}$, and from the overdamped equation of motion, $\mathbf{0}=-\zeta \boldsymbol{U}(t)+$ $\boldsymbol{F}(t)$. We obtain $\boldsymbol{\sigma}=-n\langle\boldsymbol{x} \boldsymbol{F}\rangle=-n \zeta \int\left\langle\boldsymbol{U}\left(t^{\prime}\right) \boldsymbol{U}(t)\right\rangle d t^{\prime}=$ $-n \zeta \boldsymbol{D}$, where the time integral of the velocity autocorrelation is the diffusivity of the particle, $\boldsymbol{D}$. A particle undergoing any type of random motion therefore exerts a pressure $\Pi=-\operatorname{tr} \sigma / 3=n \zeta D$. This general result applies for an 
arbitrary particle shape (where $\zeta$ may depend on particle configuration) and for any source of random motion. Indeed, it has been used in the context of microrheology [6]. It applies equally well to Brownian particles where $D=k_{B} T / \zeta$, and we obtain the familiar ideal-gas Brownian osmotic pressure $\Pi^{B}=n k_{B} T$. Using the convective diffusivity of dilute active matter, $D=U_{0}^{2} \tau_{R} / 6$ [7], we arrive at the analogous "ideal-gas" swim pressure,

$$
\Pi^{\mathrm{swim}}(\phi \rightarrow 0)=n \zeta U_{0}^{2} \tau_{R} / 6,
$$

where $\phi=4 \pi a^{3} n / 3$ is the volume fraction of active particles. As expected for dilute systems, $\Pi^{\text {swim }}$ depends on the particle size only through the hydrodynamic drag factor $\zeta$ and is entirely athermal in origin. In two dimensions, $\Pi^{\text {swim }}=n \zeta U_{0}^{2} \tau_{R} / 2$. From Eq. (1) the swim pressure is the average force moment, with $\zeta U_{0}$ the force and the moment arm is the run length in a reorientation time, $U_{0} \tau_{R}$.

To verify the existence of a swim pressure, we conducted Brownian dynamics (BD) simulations with active particles placed inside a simulation cell both with and without bounding walls. For now we focus on non-Brownian particles (no translational diffusion) to verify that this pressure arises solely from self-propulsion. The system was evolved following the $N$-particle Langevin equation, $\mathbf{0}=-\zeta\left(\boldsymbol{U}-\boldsymbol{U}_{0}\right)+\boldsymbol{F}^{P}$ and $\mathbf{0}=-\zeta_{R} \Omega+\boldsymbol{L}^{R}$, where $\boldsymbol{U}$ and $\Omega$ are the translational and angular velocities, $\zeta_{R}$ is the hydrodynamic resistance coupling angular velocity to torque, $\boldsymbol{F}^{P}$ is a hard-sphere interparticle force that prevents particle overlaps computed from a potential-free algorithm $[8,9]$, and $\boldsymbol{L}^{R}$ is the reorientation torque. The left-hand side is zero because inertia is negligible for colloidal dispersions.

In the absence of interparticle forces $\left(\boldsymbol{F}^{P}=\mathbf{0}\right)$, active swimming is a force-free motion, $\zeta\left(\boldsymbol{U}-\boldsymbol{U}_{0}\right)=\mathbf{0}$, giving $\boldsymbol{U}=\boldsymbol{U}_{0}$. However, it is both permissible, and essential for computing the stress, to interpret $\zeta \boldsymbol{U}_{0}$ as a swim force, i.e., $\boldsymbol{F}^{\text {swim }} \equiv \zeta \boldsymbol{U}_{0}$, where $\boldsymbol{U}_{0}=U_{0} \boldsymbol{q} ; U_{0}$ is the swimming speed and $\boldsymbol{q}$ is the unit orientation vector of the swimmer. The active particle velocity then follows from $\boldsymbol{U}=\boldsymbol{F}^{\text {swim }} / \zeta=\boldsymbol{U}_{0}$. One way to appreciate the swim force is to suppose that we prevent an active swimmer from moving, say by optical tweezers. The force required to hold the swimmer fixed is precisely $\zeta \boldsymbol{U}_{0}$. This use of the swim force to compute the stress does not imply that the selfpropulsive motion generates a long-range $(1 / r)$ hydrodynamic velocity disturbance typical of low Reynolds number flows.

The reorientation torque has the white noise statistics $\overline{\boldsymbol{L}^{R}}=\mathbf{0}$ and $\overline{\boldsymbol{L}^{R}(0) \boldsymbol{L}^{R}(t)}=2 \zeta_{R}^{2} \delta(t) \boldsymbol{I} / \tau_{R}$. Particle orientations were updated by relating $\Omega$ to the instantaneous orientation $\boldsymbol{q}$ [10]. Simulations were conducted with 2000 particles for at least $5 \tau_{R}$. We varied the volume fraction $\phi$ and the nondimensional reorientation Péclet number $\mathrm{Pe}_{R}=U_{0} a / D=U_{0} a /\left(U_{0}^{2} \tau_{R}\right)=a /\left(U_{0} \tau_{R}\right)$, which is also

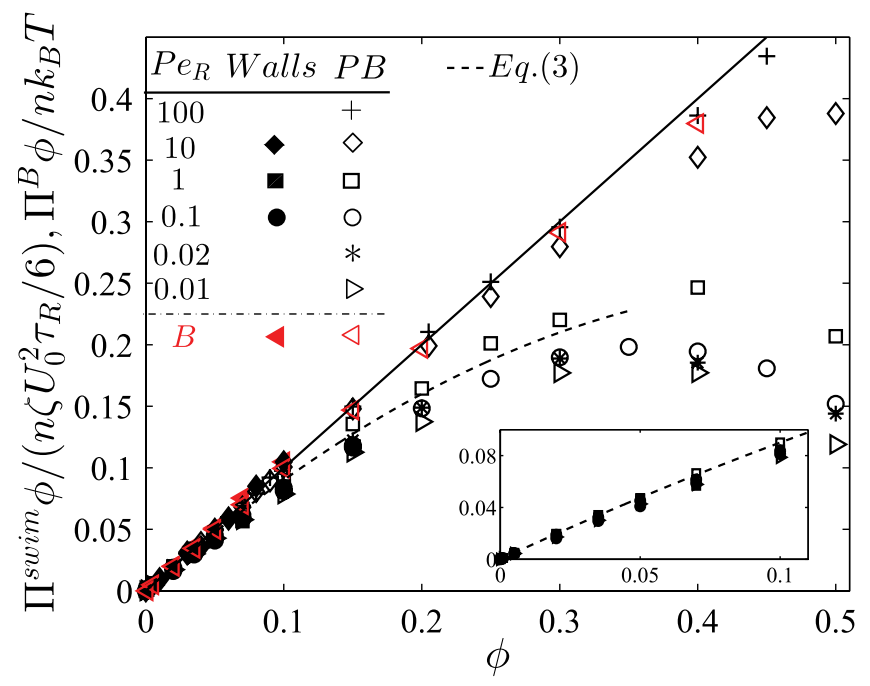

FIG. 1 (color online). The swim, $\Pi^{\text {swim }}$, and Brownian, $\Pi^{B}$, pressures computed using bounding walls ("Walls") and from Eq. (1) without walls (periodic boundaries, "PB") for various $\mathrm{Pe}_{R}=a /\left(U_{0} \tau_{R}\right)$. The solid black line corresponds to a linear increase of pressure with $\phi$. The dashed curve is the dilute theory expression [Eq. (3)]. The inset is a magnification of the swim pressure at dilute $\phi$ for $\mathrm{Pe}_{R} \leq 1$.

the ratio of the particle size $a$ to the run length of the active particles, $U_{0} \tau_{R}$ [11]. The pressure was obtained by dividing the force, calculated from the hard-sphere displacements at the wall [9], by its area, $F^{\text {wall }} / A$. In addition, we conducted BD simulations using periodic boundaries (without bounding walls) and used Eq. (1) to compute the swim pressure. For a wide range of $\mathrm{Pe}_{R}(=0.01-100)$ and small $\phi$, all data collapse onto the predicted pressure, $\Pi^{\mathrm{swim}}=n \zeta U_{0}^{2} \tau_{R} / 6$ (see Fig. 1). This verifies the existence of a unique swim pressure of active matter.

The concept of confinement also applies for purely Brownian particles, where the wall pressure in this case is $\Pi^{B}=n k_{B} T$. Figure 1 shows the osmotic pressure of purely Brownian particles alongside the swim pressure of active matter. For active swimmers, the data (black symbols) collapse onto the line $\Pi^{\text {swim }}=n \zeta U_{0}^{2} \tau_{R} / 6$ until around $\phi \sim 0.1$. At higher $\phi$, the swim pressure decreases because the particles collide and obstruct each others' movement for a time $\tau_{R}$ until their swimming directions change from rotational motion. This clustering behavior [12] reduces the average distance they travel between reorientations and thus reduces the diffusivity and pressure (by reducing the moment arm). This differentiates active matter from an equilibrium Brownian system, which exerts a fixed $\Pi^{B}=$ $n k_{B} T$ of ideal-gas pressure for all $\phi$. For high $\mathrm{Pe}_{R}$ (small $\tau_{R}$ ), the active particles reorient rapidly with small swim steps and behave as random walkers, so no clusters form and $\Pi^{\text {swim }}$ increases linearly with $\phi$ until very high $\phi(\sim 0.6)$.

The dashed curve in Fig. 1 is our theoretical prediction of the swim pressure for small $\phi$ using results from nonlinear microrheology [13]. The microrheological probe particle is a 
swimmer that is propelled through a colloidal dispersion with a constant swim force. Active microrheology describes our system accurately for high $\mathrm{Pe}_{s}=F^{\text {swim }} /\left(k_{B} T / a\right)$ and a large reorientation time compared to the collisional time, i.e., $\tau_{R}>a /\left(U_{0} \phi\right)$, or $\phi>a /\left(U_{0} \tau_{R}\right)=\mathrm{Pe}_{R}$. This ensures that a steady-state microstructure is achieved during the course of the swimmer's run length. Substituting the high- $\mathrm{Pe}_{s}$ results from Squires and Brady [13] (Eq. (36) of their paper) into Eq. (1), we obtain the leading-order correction to the idealgas swim pressure,

$$
\frac{\Pi^{\text {swim }}}{n \zeta U_{0}^{2} \tau_{R} / 6}=1-\phi+\mathcal{O}\left(\phi^{2}\right)
$$

which agrees well with our BD simulations for $\phi \lesssim 0.1$ (see inset of Fig. 1). In contrast to Brownian systems where repulsive pair interactions (e.g., excluded volume) always increase the pressure, for active matter interactions decrease the run length and therefore the swim pressure.

The total pressure of active matter (in the absence of hydrodynamic interactions) is given by $P=p_{f}+\Pi^{\text {act }}$, where $\Pi^{\text {act }}=\Pi^{\text {swim }}+\Pi^{P}, \quad p_{f}$ is the solvent pressure, which is arbitrary for an incompressible fluid (plays no dynamical role and is set to zero), and $\Pi^{P}$ is the pressure due to interparticle (e.g., excluded volume) forces, $\boldsymbol{\sigma}^{P}=-n\left\langle\boldsymbol{x} \boldsymbol{F}^{P}\right\rangle$. Following Brady [14], we computed the analytical expression for $\Pi^{P}$ for hard-sphere particles using the results from nonlinear microrheology: $\Pi^{P} /$ $\left(n \zeta U_{0}^{2} \tau_{R} / 6\right)=3 \mathrm{Pe}_{R} \phi+\mathcal{O}\left(\phi^{2}\right)$. Unlike the swim pressure, the interparticle pressure scales as $n^{2} \zeta U_{0} a^{4}$ since the particle size now sets the scale for the force moment. This is different from $\Pi^{\text {swim }} \sim n \zeta U_{0}\left(U_{0} \tau_{R}\right)$, which is a single-particle contribution where the run length $U_{0} \tau_{R}$ sets the scale for the force moment.

Combining this result with Eq. (3), we obtain a nonequilibrium virial equation of state for active matter,

$$
\frac{\Pi^{\text {act }}}{n \zeta U_{0}^{2} \tau_{R} / 6}=1-\phi\left(1-3 \mathrm{Pe}_{R}\right)+\mathcal{O}\left(\phi^{2}\right)
$$

which can be rewritten as $\Pi^{\text {act }} /\left(n \zeta U_{0}^{2} \tau_{R} / 6\right)=1+$ $B_{2} n+\cdots$, where $B_{2}=-\pi(2 a)^{3}\left(1-3 \mathrm{Pe}_{R}\right) / 6$ is analogous to the second-virial coefficient from a classical thermodynamic system and is negative for $\mathrm{Pe}_{R}<1 / 3$. The reorientation Péclet number $\mathrm{Pe}_{R}=a /\left(U_{0} \tau_{R}\right)$ is analogous to the temperature in a classical equation of state. If $\mathrm{Pe}_{R} \gg 1$, then the particle takes small random steps and behaves like a Brownian walker. If $\mathrm{Pe}_{R} \ll 1$, then the reorientation time is large, causing the particles to obstruct each others' paths when they collide and reduce their run lengths.

We now apply this new swim stress perspective to analyze self-assembly and phase separation in active soft matter. Experiments and computer simulations [12,15-20] have shown that active matter self-organizes into dense and dilute phases resembling an equilibrium liquid-gas

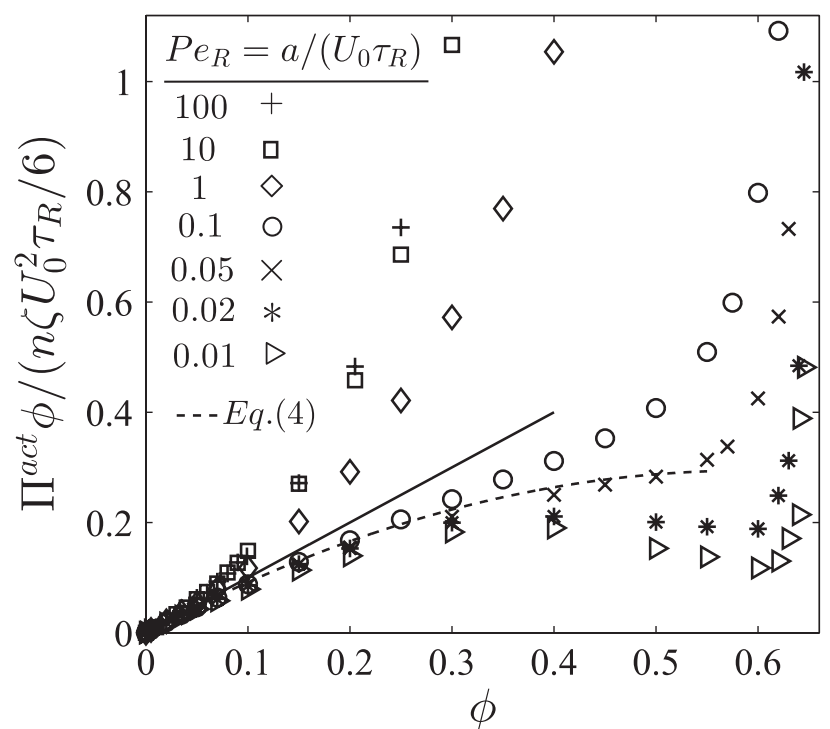

FIG. 2. Nonequilibrium $\Pi^{\text {act }}-\phi$ phase diagram, where $\Pi^{\text {act }}=\Pi^{\text {swim }}+\Pi^{P}$. The data are from BD simulations (with periodic boundaries) and the dashed curve is the analytical theory, Eq. (4), with $\mathrm{Pe}_{R}=a /\left(U_{0} \tau_{R}\right)=0.05$.

coexistence. Continuum descriptions [19,21] and other microscopic approaches [17,18,21-23] have provided models for this behavior, but the question remains as to whether there is a simpler and more primitive explanation for the self-assembly in active matter.

From classical thermodynamics, a negative second virial coefficient $B_{2}$ (i.e., two-body attractions) implies the possibility of a gas-liquid phase transition. Attractions may give rise to a nonmonotonic variation of pressure with concentration, known as a van der Waals loop. Here we show that our simple active system has a pressure-volume phase diagram much like that of a van der Waals fluid.

We conducted BD simulations (with periodic boundary conditions) to produce the full $\Pi^{\text {act }} \phi \phi$ phase diagrams for different values of $\mathrm{Pe}_{R}$. The system was initialized by placing the active particles at random configurations inside the simulation cell. For concentrated systems, we used a modified Lubachevsky-Stillinger algorithm [24]. At each time step, we followed Foss and Brady [9] to compute the interparticle stresslet $-\left\langle\boldsymbol{x} \boldsymbol{F}^{P}\right\rangle$. The swim stresslet $-\left\langle\boldsymbol{x} \boldsymbol{F}^{\text {swim }}\right\rangle$ was computed by correlating the positions of the particles with their swim force. The particle positions $x$ are continuous across the periodic boundaries when computing $-\left\langle\boldsymbol{x} \boldsymbol{F}^{\text {swim }}\right\rangle$.

As shown in Fig. 2, at low $\phi$ all data collapse onto the ideal-gas pressure given by Eq. (2). At high $\mathrm{Pe}_{R}$ (high "temperature"), the interparticle pressure dominates and the total pressure increases monotonically with $\phi$. This corroborates with Eq. (4), which gives a positive $B_{2}$ for $\mathrm{Pe}_{R}>1 / 3$. As in classical thermodynamics, phase separation is possible when $\mathrm{Pe}_{R}$ (the "temperature") is below the critical point $\left(\partial \Pi^{\text {act }} / \partial \phi=\partial^{2} \Pi^{\text {act }} / \partial \phi^{2}=0\right)$, beyond which the system is 


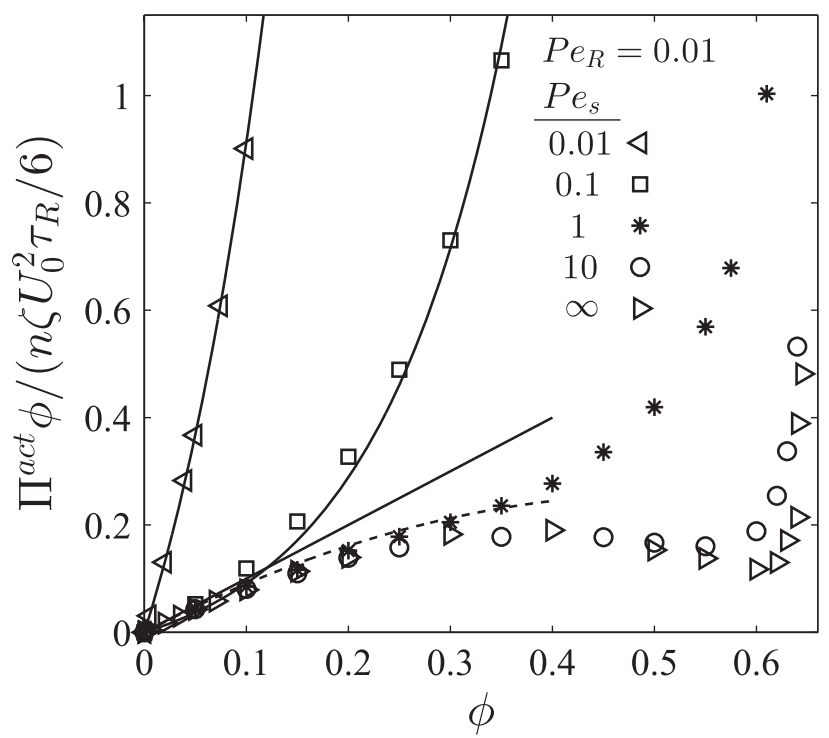

FIG. 3. Effect of translational Brownian motion on the $\Pi^{\text {act }}-\phi$ phase diagram, where $\Pi^{\text {act }}=\Pi^{\text {swim }}+\Pi^{P}+\Pi^{B}$. The data are BD simulations for various $\mathrm{Pe}_{s}=U_{0} a / D_{0}$ with fixed $\mathrm{Pe}_{R}=$ $a /\left(U_{0} \tau_{R}\right)=0.01$. The solid curves are theoretical expressions of osmotic pressures of Brownian particles. The dashed curve is the analytical theory, Eq. (4).

supercritical. As $\mathrm{Pe}_{R}$ is reduced below $\sim 0.03$, we observe a nonmonotonic pressure profile resembling a van der Waals loop-the total pressure is lower at $\phi=0.6$ than at $\phi=0.3$. This decrease is caused by the reduction in swim pressure due to the particles' tendency to form clusters, reducing the average distance they travel between reorientations, i.e., reducing the moment arm $\boldsymbol{U} \tau_{R}$. A study of structural properties [25] corroborates with our nonmonotonic pressure profiles. The probability distribution of the local volume fraction of swimmers computed using a Voronoi construction becomes bimodal at low $\mathrm{Pe}_{R}$ and finite $\phi$, indicating the presence of a dilute and dense phase. As $\phi$ approaches close packing, $\Pi^{\text {swim }} \rightarrow 0$ but the total pressure necessarily increases because the interparticle (excluded volume) pressure diverges to infinity.

We now consider the effects of translational Brownian motion, which affects the interparticle pressure and contributes the dilute Brownian osmotic pressure to the active pressure: $\quad \Pi^{\text {act }}=\Pi^{\text {swim }}+\Pi^{P}+\Pi^{B}$. The system also involves the swim Péclet number, $\mathrm{Pe}_{s}=F^{\text {swim }} /\left(k_{B} T / a\right)=$ $U_{0} a / D_{0}$, where $D_{0}=k_{B} T / \zeta$ is the Stokes-EinsteinSutherland diffusivity of an isolated Brownian particle. Physically, translational Brownian motion would tend to restore the phase-separated system back to a homogeneous state. We would no longer see nonmonotonic pressure profiles as $\mathrm{Pe}_{s}$ is reduced from our non-Brownian $\left(\mathrm{Pe}_{s} \rightarrow \infty\right)$ system, and the $\Pi^{\text {act }}-\phi$ curve would approach the pure Brownian limit as $\mathrm{Pe}_{s} \rightarrow 0$. Indeed, these qualitative predictions are corroborated by Fig. 3, where we conducted simulations with active particles that translate by both self-propulsion and translational diffusion: $\mathbf{0}=-\zeta \boldsymbol{U}+$ $\boldsymbol{F}^{\text {swim }}+\boldsymbol{F}^{B}+\boldsymbol{F}^{P}$, and the Brownian force has the usual properties $\overline{\boldsymbol{F}^{B}}=\mathbf{0}$ and $\overline{\boldsymbol{F}^{B}(0) \boldsymbol{F}^{B}(t)}=2 k_{B} T \zeta \delta(t) \boldsymbol{I}$. As shown in Fig. 3, increasing the translational Brownian motion (decreasing $\mathrm{Pe}_{s}$ ) causes the van der Waals loop to vanish and the pressure approaches the familiar osmotic pressure of Brownian particles, i.e., a monotonic increase with volume fraction. The solid curves for $\mathrm{Pe}_{s}=0.1$ and 0.01 correspond to the theoretical prediction of Brownian osmotic pressure [14] from the Carnahan-Starling equation of state, scaled appropriately by each $\mathrm{Pe}_{s}$.

Self-propelled particles need not be spherical nor have a constant intrinsic velocity $\boldsymbol{U}_{0}$ or reorientation time $\tau_{R}$. We neglected hydrodynamic interactions between particles, which would contribute additional terms to the pressure and affect the reorientation time. The swim pressure is distinct from, and in addition to, the "hydrodynamic stresslet" that accompanies a class of nonspherical microswimmers [26,27], which scales as $n \zeta U_{0} a(\boldsymbol{q} \boldsymbol{q}-\boldsymbol{I} / 3)$ and averages to zero for an isotropic distribution. The ratio of the magnitude of the hydrodynamic stresslet over the swim pressure is $\mathrm{Pe}_{R}$; the hydrodynamic contribution becomes negligible when phase separation occurs at low $\mathrm{Pe}_{R}$ (see Fig. 2).

We have allowed for any reorientation mechanism characterized solely by $\tau_{R}$. If the reorientation is caused by thermal Brownian rotations, then $\tau_{R}=1 / D_{R}$, where the rotary diffusivity $D_{R}=k_{B} T / \zeta_{R}=k_{B} T /\left(8 \pi \eta a^{3}\right)$ for an isolated sphere. In this case our $\mathrm{Pe}_{R}=a D_{R} / U_{0}=3 /\left(4 \mathrm{Pe}_{s}\right)$ and so the dual limits for phase separation $\mathrm{Pe}_{R} \rightarrow 0, \mathrm{Pe}_{s} \rightarrow \infty$ are automatically satisfied. Indeed, the ratio of the Brownian osmotic pressure to the active swim pressure $\Pi^{B} / \Pi^{\text {swim }}=$ $k_{B} T /\left(\zeta U_{0}^{2} \tau_{R} / 6\right)=6 \mathrm{Pe}_{R} / \mathrm{Pe}_{s} \ll 1$ for possible phase separation, in agreement with the results in Fig. 3.

Through their self-motion, all active matter systems generate a swim pressure. The quantitative prediction of phase separation is one of many applications of the swim stress perspective. A possible application is the analysis of various biophysical systems, such as the crowded interior of a cell. For example, motor proteins moving along a microtubule will exert a tension along the tube simply because they are confined to the tube. Another application is the development of soft materials that exploit the selforganization behavior of active matter, which includes a class of artificial catalytic motors [15,16,28].

This swim pressure exists at all scales in both living (e.g., microorganisms) and nonliving active systems, and also applies to larger swimmers (e.g., fish) where inertia is important (i.e., the Reynolds number is not small). This swim stress is in addition to the usual Reynolds stress contribution $\rho\left\langle\boldsymbol{u}^{\prime} \boldsymbol{u}^{\prime}\right\rangle$, where $\rho$ is the density and $\boldsymbol{u}^{\prime}$ is the velocity fluctuation. Experimental measurement of the swim pressure requires the confining boundaries to be permeable to the solvent, just as is the case for the osmotic pressure of a solute. 
Lastly, one can construct a flux model from our swim pressure perspective. The conservation of active particle number density is $\partial n / \partial t+\nabla \cdot \boldsymbol{j}=0$, where $\boldsymbol{j}=n \boldsymbol{v}$ is the particle flux and $\boldsymbol{v}$ is the average velocity of the particles. Standard volume averaging gives a momentum balance (for negligible inertia) relating the particle flux to stress gradients: $\mathbf{0}=-\zeta n(\boldsymbol{v}-\boldsymbol{u}) /(1-\phi)+\nabla \cdot \boldsymbol{\sigma}^{\text {act }}$, where $\boldsymbol{\sigma}^{\text {act }}=$ $\boldsymbol{\sigma}^{\text {swim }}+\boldsymbol{\sigma}^{P}$ and $\boldsymbol{u}$ is the volume-average velocity of the suspension. The relative flux $\boldsymbol{j}_{\text {rel }}=n(\boldsymbol{v}-\boldsymbol{u})=$ $(1-\phi) \nabla \cdot \sigma^{\text {act }} / \zeta$, and the conservation of particle number density becomes an advection-diffusion equation with $\boldsymbol{D}=-(1-\phi)\left(\partial \boldsymbol{\sigma}^{\text {act }} / \partial n\right) / \zeta$, which recovers $\boldsymbol{D}=U_{0}^{2} \tau_{R} \boldsymbol{I} / 6$ for dilute active matter. For a thermodynamic system (slightly) out of equilibrium the driving force for motion is the gradient in the chemical potential $\nabla \mu$. From the active particle momentum balance we obtain $n(\partial \mu / \partial n)=$ $(1-\phi) \partial \Pi^{\text {act }} / \partial n$, which may provide the necessary generalization of the chemical potential for nonequilibrium active matter.

S. C. T is supported by the Gates Millennium Scholars fellowship and the National Science Foundation Graduate Research Fellowship under Grant No. DGE-1144469.

*jfbrady@caltech.edu

[1] D. J. Sumpter, Collective Animal Behavior (Princeton University, Princeton, NJ, 2010).

[2] S. Ramaswamy, Annu. Rev. Condens. Matter Phys. 1, 323 (2010).

[3] J. Toner, Y. Tu, and S. Ramaswamy, Ann. Phys. (Amsterdam) 318, 170 (2005).

[4] Indeed, Brady [14] showed that there is no need to restrict the interaction to be pairwise, and Eq. (1) is compatible with the traditional micromechanical definition of stress.

[5] H. Goldstein, Classical Mechanics (Addison-Wesley, Reading, MA, 1990), 2nd ed.

[6] R. N. Zia and J. F. Brady, J. Rheol. 56, 1175 (2012).

[7] H. Berg, Random Walks in Biology (Princeton University, Princeton, NJ, 1993).
[8] D. M. Heyes and J. R. Melrose, J. Non-Newtonian Fluid Mech. 46, 1 (1993).

[9] D. R. Foss and J. F. Brady, J. Rheol. 44, 629 (2000).

[10] D. A. Beard and T. Schlick, Biophys. J. 85, 2973 (2003).

[11] We use the conventional definition of the Péclet number as advection over diffusion, but others use the inverse of this quantity.

[12] M.E. Cates and J. Tailleur, Europhys. Lett. 101, 20010 (2013).

[13] T. M. Squires and J. F. Brady, Phys. Fluids 17, 073101 (2005).

[14] J. F. Brady, J. Chem. Phys. 98, 3335 (1993).

[15] I. Theurkauff, C. Cottin-Bizonne, J. Palacci, C. Ybert, and L. Bocquet, Phys. Rev. Lett. 108, 268303 (2012).

[16] J. Palacci, S. Sacanna, A. P. Steinberg, D. J. Pine, and P. M. Chaikin, Science 339, 936 (2013).

[17] G. S. Redner, M. F. Hagan, and A. Baskaran, Phys. Rev. Lett. 110, 055701 (2013).

[18] J. Bialké, H. Löwen, and T. Speck, Europhys. Lett. 103, 30008 (2013).

[19] J. Stenhammar, A. Tiribocchi, R. J. Allen, D. Marenduzzo, and M. E. Cates, Phys. Rev. Lett. 111, 145702 (2013).

[20] M. E. Cates, D. Marenduzzo, I. Pagonabarraga, and J. Tailleur, Proc. Natl. Acad. Sci. U.S.A. 107, 11715 (2010).

[21] Y. Fily and M. C. Marchetti, Phys. Rev. Lett. 108, 235702 (2012).

[22] R. Ni, M. A. C. Stuart, and M. Dijkstra, Nat. Commun. 4, 2704 (2013).

[23] A. Baskaran and M. C. Marchetti, Phys. Rev. Lett. 101, 268101 (2008).

[24] M. Skoge, A. Donev, F. H. Stillinger, and S. Torquato, Phys. Rev. E 74, 041127 (2006).

[25] A. Wysocki, R. G. Winkler, and G. Gompper, Europhys. Lett. 105, 48004 (2014).

[26] D. Saintillan and M. J. Shelley, Phys. Fluids 20, 123304 (2008).

[27] T. Ishikawa, M. P. Simmonds, and T. J. Pedley, J. Fluid Mech. 568, 119 (2006).

[28] U. M. Córdova-Figueroa and J. F. Brady, Phys. Rev. Lett. 100, 158303 (2008). 